\title{
Étude rétrospective de 24 cas de métastases bucco-faciales issus de deux Centres de lutte contre le cancer et synthèse de la littérature
}

\author{
Maschino F, Curien R, Sourdot A, Lê A, Bally J, Bravetti P \\ UFR d'Odontologie, Département de Chirurgie buccale, Nancy, France \\ francois.maschino@free.fr
}

Les métastases dans la cavité buccale et les os de la face sont considérées comme rares. Elles représentent 1 à 3,2\% (Meyer 1965, Zachariades 2004, Hirshberg 2008), voire 5\% (Pindborg 1995), des tumeurs malignes de cette région. Les signes cliniques peu spécifiques, l'aspect faussement bénin et le caractère souvent isolé de la métastase sont autant de pièges diagnostiques que le clinicien doit savoir éviter. Ces métastases sont souvent mal identifiées, ce qui diffère d'autant un diagnostic important pour le patient.

Les métastases dans les tissus mous sont souvent confondues avec un granulome pyogénique, une épulis fibreuse, un granulome à cellules géantes, un hémangiome ou un fibrome périphérique (Micali 1994, Rim 2003). Il est parfois difficile de les suspecter notamment lorsqu'elles sont inaugurales. Elles sont parfois évocatrices, simulant un carcinome épidermoïde ou un lymphome (Vieira et al. 2001). Celles des os de la face peuvent mimer un kératokyste, une ostéite ou une ostéomyélite, une tumeur osseuse bénigne (améloblastome), un granulome à cellules géantes, et parfois une tumeur maligne primitive tel qu'un ostéosarcome, un myélome multiple, une tumeur parotidienne ou un carcinome épidermoïde lorsque la métastase s'extériorise (Meyer 1965, Rohrer 1981).

Les signes cliniques sont assez souvent discrets et peu évocateurs : tuméfaction asymptomatique, douleurs pouvant orienter vers une origine odontogénique, un SADAM... Cependant la croissance rapide, la perte spontanée de dents ou la présence d'une paresthésie doit attirer l'attention du clinicien.

Une étude rétrospective a été réalisée pour analyser les cas de métastases bucco-faciales trouvés dans les centres de lutte contre le cancer de Nancy et de Dijon et de les comparer aux données de la littérature en langues anglaise, française et allemande.

Cette étude comprend 24 patients (16 hommes, 8 femmes), âgés de 32 à 84 ans (âge moyen : 64,3 ans), pris en charge au Centre Alexis Vautrin (Nancy) ou au Centre Georges François Leclerc (Dijon) entre 1981 et 2008 pour le traitement d'un cancer et qui ont développé une métastase dans la sphère bucco-faciale, avec un examen histologique ayant permis d'identifier la tumeur primitive. Cinq de ces patients ont présenté une métastase inaugurale $(20,8 \%)$; le délai d'apparition de la métastase pour les autres patients a varié de 0 (découverte synchrone) à 316 mois après le diagnostic du cancer (délai moyen : 32 mois). La survie après le diagnostic de la métastase a été de 1 à 63 mois (délai moyen : 14,1 mois) et le taux de survie à 1 an et à 2 ans a été respectivement de $50 \%$ et de $29,1 \%$. Les cancers primitifs les plus fréquemment retrouvés sont d'origine pulmonaire ( 7 cas), rénale ( 4 cas), mammaire ( 2 cas) et thyroïdienne ( 2 cas). Six métastases affectaient les os (3 à la mandibule et 3 le maxillaire) et 18 cas les tissus mous (10 la gencive et la muqueuse alvéolaire, 5 la langue, 1 la parotide et 3 le voile du palais et ses piliers). 